\title{
Integrating Mid-Air Haptics into Movie Experiences
}

\author{
Damien Ablart ${ }^{\dagger}$, Carlos Velasco ${ }^{\ddagger}$, Marianna Obrist ${ }^{\dagger}$ \\ $\dagger$ SCHI Lab, School of Engineering and Informatics, University of Sussex, UK \\ †Department of Marketing, BI Norwegian Business School \\ da292@sussex.ac.uk, carlos.velasco@bi.no,m.obrist@sussex.ac.uk
}

\begin{abstract}
"Seeing is believing, but feeling is the truth". This idiom from the seventieth century English clergyman Thomas Fuller gains new momentum in light of an increased proliferation of haptic technologies that allow people to have various kinds of 'touch' and 'touchless' interactions. Here, we report on the process of creating and integrating touchless feedback (i.e. mid-air haptic stimuli) into short movie experiences (i.e. one-minute movie format). Based on a systematic evaluation of user's experiences of those haptically enhanced movies, we show evidence for the positive effect of haptic feedback during the first viewing experience, but also for a repeated viewing after two weeks. This opens up a promising design space for content creators and researchers interested in sensory augmentation of audiovisual content. We discuss our findings and the use of mid-air haptics technologies with respect to its effect on users' emotions, changes in the viewing experience over time, and the effects of synchronisation.
\end{abstract}

\section{ACM Classification Keywords}

H.5.m. Information Interfaces and Presentation (e.g. HCI).

\section{Author Keywords}

Mid-Air Haptic; Touch; User Experience; Movie Experiences; Skin Conductance Response; Short Movie; Emotions.

\section{INTRODUCTION}

Audiovisual media has become omnipresent in people's everyday lives and has a significant impact on their feelings and emotions [2,19]. Over the last few years, the sense of touch has gained attention as a means to enhance users' experiences, particularly to create more immersive media experiences. For example, Surround Haptics provides smooth tactile motions on the back through a system that is integrated in a seat [13], a tactile jacket that triggers vibrations to intensify emotions [16], AIREAL uses vortexes of air that delivers tactile sensations in free air [28], and Ultrahaptics that display ultrasonic waves to create tactile sensations in mid-air [5]. The first two examples require physical contact, while the latter two generate tactile sensations in the air, not requiring any physical contact between the user and the interface.

Permission to make digital or hard copies of all or part of this work for personal or classroom use is granted without fee provided that copies are not made or distributed for profit or commercial advantage and that copies bear this notice and the full citation on the first page. Copyrights for components of this work owned by others than ACM must be honored. Abstracting with credit is permitted. To copy otherwise, or republish, to post on servers or to redistribute to lists, requires prior specific permission and/or a fee. Request permissions from permissions@acm.org.

TVX 2017, June 14-16, 2017, Hilversum, The Netherlands.

Copyright (C) 2017 ACM ISBN 978-1-4503-4529-3/17/06 ...\$15.00.

http://dx.doi.org/10.1145/3077548.3077551
In this paper, we focus on mid-air haptic technology and its effect on media experiences, as it has not been studied before. More precisely we focus on mid-air haptic feedback and their potential role in movie experiences. There is a growing body of knowledge on the perception of mid-air haptic stimuli (localisation and discrimination) [31] and the creation of shapes in mid-air [18]. However, the effects of these kinds of stimulation on human emotions has only recently been studied.

In contrast to previous studies where the haptic experience is created to match a specific emotion [16], to mirror the screen [15], or to match the specific semantic space [13], we designed a single haptic pattern to enhance viewers' experiences. By pattern, we mean a mid-air haptic creation defined by an intensity, a movement, and a frequency over time. We explored this pattern with respect to its temporal integration into movies (synchronized versus not synchronized with the peak moments in a movie). We focus on "one-minute movies", which is a content format that conveys a complete narrative in one minute and allows a comparable set of movies of the same format and length. Then, we conducted a study following three main steps: (1) selection of movies, (2) creation and integration of haptic feedback (haptic pattern) into the movie narrative (synchronised vs not synchronized) and (3) evaluation of the users' viewing experiences (emotions) in two instances (two weeks separated). For the evaluation, we used three conditions: (a) with and without haptic feedback, (b) movie-specific design versus one cross-movies design, and (c) repeated viewing after two weeks. We used a combination of measures (i.e. self-report questionnaires and skin conductance responses) to capture the effect of the haptic feedback on users viewing experiences.

The present study contributes to the growing literature of haptic experience [27] and multisensory experience design [25]. First, we demonstrate the integration of mid-air haptic feedback into audiovisual content in form of a simple haptic pattern. This approach can be further extended towards a variety of pre-defined and custom-made or even automated patterns in the future. Second, we describe a methodological procedure to study the immediate and more long-term effect of haptic feedback. Finally, we discuss future directions for research, and possible developments in the broader context of media experiences.

\section{RELATED WORK}

In this section, we discuss relevant previous work that has explored the potential of the senses to enhance movie experiences. We first present an overview of the media and the 
senses and we then focus on the use of mid-air haptics and the challenges of designing haptic feedback for one-minute movies.

The senses (i.e. smell, taste, and touch) are a relevant component of Human-Computer Interaction [24] and have been studied in the context of interactive media [29]. The MPEG-V ISO standard [12] and Mulsemedia [8] are good examples of the effort made to create standards for the multisensory integration into media.

The sense of smell has been studied with media, in a recent survey Murray [20] exposes various context of olfactory integration with media. On the other hand, the sense of taste has received little attention but recent works $[21,26]$ show interesting new interaction mechanisms that could open new ways of integrating taste with media. The sense of touch is presented in the next section.

\section{Haptically enhanced media experiences}

Touch is a powerful means to communicate emotions [10]. Indeed, researchers have aimed to reproduce its richness in haptic feedback system. Simple examples of such systems include vibrations of our mobile phones [30], video game controllers [7], and force feedback in steering wheels for racing games [11]. More specifically, Israr et al. [13] introduced the idea of Surround Haptics, a new tactile technology that uses a low-resolution grid of vibrating actuators to generate highresolution, continuous, moving tactile strokes on the human skin. Different game events are mapped to different haptic feedback patterns. Those patterns are sent to the user through a chair embedded with vibratory actuators on the back. This is an interesting example of more immersive experiences that is based on a carefully designed video-tactile-audio gaming environment.

While the previous example of Surround Haptics requires actual physical contact with the user, new haptic technologies that promote the idea of touchless interaction for media experiences have emerged over the last years. Sodhi et al. [28], for example, developed AIREAL, a haptic technology that delivers tactile sensations in free air using vortex-based tactile actuation. An air vortex is a ring of air that can travel at high speeds over larger distances to create free air haptic experiences.

In the present research, we are particularly interested in midair haptic technology presented by Shinoda et al. [1], the only mid-air technology that allows the creation of real-time patterns with various frequencies and intensity. It is composed of a series of ultrasonic transducers that emit very high frequency sound waves. When all of the sound waves meet at the same location at the same time, they stimulate the human's skin creating haptic sensations in mid-air. No gloves or attachments to the user's body are required as the feeling is directly projected onto the user's hands (or body part).

Previous work using this mid-air haptic technology has provided insights into the perception and localisation of mid-air haptic stimuli [31], the creation of complex haptic patterns such as shapes [18], and most recently the mediation of emotions through mid-air haptics [23]. The challenge is still to understand how to create the right haptic experience for a given media or movies.

\section{Designing tactile experiences for movies}

Various approaches have been explored to design haptic feedback for movies. Danieau et al. [6], for instance, recorded haptic feedback experienced during specific activities (e.g. horse riding) alongside video and sound. Users experienced the movies with 3 different haptic conditions (recorded, randomly generated, and no haptic feedback) and rated them using a Quality of Experience (QoE) questionnaire. Users rated the captured haptic feedback as more immersive than random haptic feedback and the random feedback was also better than no-feedback at all. While those findings are interesting, this approach is mainly focusing on the mirroring of an action (motion) on the screen and hence the stimulation of the visual sense, rather than the sense of touch.

Lemmens et al. [16], in contrast, created patterns for a haptic jacket based on typical touch behaviours from human emotional touch communication (e.g. highly energetic movements to indicate surprise or happiness) as well as based on common wisdoms and sayings (e.g. butterflies in your stomach). Those patterns were presented together with short movies. Users reactions were assessed through physiological measurements (respiration, heart rate, skin conductance level) and questionnaires (SAM [4] and Immersion Questionnaire). The results suggested a positive effect of haptic stimuli on peoples' immersion but they used only one haptic condition per movies, making any comparison between the designed haptics and other approaches impossible.

Israr et al. [14] proposed an approach based on a systematic exploration of haptic feedback and its integration with the other senses, as well as the content and the context of use. The authors built a library that establishes a classification between haptic feedback parameters (i.e. intensity, duration, and stimulus onset asynchrony) and semantic space (e.g. rain, pulse). This library was built and evaluated by users and can be used with various kind of media [34]. Nevertheless, there is still a need to investigate the impact of using a specific pattern during a media experience as it is very likely that the main focus will be on the visual content [9] and can thus outshine the effect of the pattern used.

More creative-focused approaches have been presented. For instance, Kim and al. [15] designed an authoring tool where users can pause a movie and draw the haptic feedback on the screen, focusing of the visual elements they judge relevant. This interface is designed to work with the haptics gloves they designed. Schneider et al. [27] extended this approach in a multi-device toolkit in order to facilitate haptic experience design. The authors designed a single interface capable of supporting various kinds of devices for creating patterns by drawing on the screen. In contrast to the toolbox approach, this toolkit might challenge designers with too many possibilities in the design of tactile experiences, especially when confronted with a new device, such as mid-air technology.

This paper expands on these previous works by designing tactile experiences using mid-air haptics technology. 


\section{STUDY}

In our research, we investigate the effect of mid-air haptic feedback on short movie experiences. We focus specifically on "one-minute movies", a content format that conveys a complete narrative in one minute and bridges traditional TV with online video consumption (e.g. YouTube). This particular format is featured in the annual "movie minute festival " that challenges movie-makers, writers, animators, artists, designers, and creative producers to develop exciting new content.

Most importantly, this "one-minute movies" format provides us with a specific comparable timeframe for our study investigating the effect of mid-air haptic feedback on viewers' experiences. The study was divided into three main steps: (1) selecting a set of one-minute movies, (2) designing the haptic feedback, and (3) evaluating the viewer experience over time. See an overview on each step in Figure 1. In the following sections, we explain each of the three steps in detail.

\section{Step 1: Selection of the one-minute movies}

The one-minute movies for our study were selected from the international one-minute movie festival collection available on YouTube. Before the first step in the user study, we selected a total of 14 one-minute movies and invited four researchers in the field of HCI to watch and rate them using the SAM. Doing so we wanted to ensure a good spread of represented movies as well as a level of agreement with respect the perceived level of valence (positive/negative) and arousal (activation) for each movie.

Each of the four invited HCI researcher was asked to watch the 14 movies and rate them according to arousal and valence using the Self-Assessment Manikin (SAM) questionnaire [4]. We also asked them to rate their liking of the movie on a 7point Likert scale ( 1 being 'didn't like it at all' to 7 'liked it a lot'). We compared the ratings for each movie and discussed them with the invited researchers with respect to the agreement on valence (if it was perceived positive, negative, or neutral) and arousal (if the movie had at least one moment of excitement, "peak moment"). The first criterion was to exclude any movies that might lead to contradicting emotional experiences and could hence be avoided for the user study. The second criterion was to inform the design of the haptic feedback along peak (arousing) moments. Based on those two criteria, two movies were excluded (one because of contradicting ratings on the valence, the other because it was perceived neutral with respect to arousal). The remaining 12 movies were used in the first step of the user study (see Figure 1).

Based on this initial pre-study step, we then recruited 22 users for our first step in the user study that lasted around $30 \mathrm{~min}$ utes and was rewarded with 6.5 USD. Each of the 22 users was invited to watch the 12 selected one-minute movies in a controlled lab environment. We used again the SAM questionnaire [4] to collect the arousal and valence ratings from users and asked them to rate their liking of each movie using the question "How much did you enjoy the movie?". We also

\footnotetext{
${ }^{1} \mathrm{http}: / /$ www.filminute.com
}

\begin{tabular}{lrr} 
one-minute movies & Valence & Arousal \\
\hline Black Hole & Neutral[0.60] & Neutral[0.58] \\
Chop Chop & Cheerful[0.71] & Neutral[0.53] \\
Grandpa & Neutral[0.47] & Neutral[0.54] \\
Loop & Sad[0.38] & High[0.63] \\
The Key & Neutral[0.63] & Low[0.41] \\
Wildebeest & Cheerful[0.73] & Neutral[0.59]
\end{tabular}

Table 1. List of the six selected movies for step two in our study - balancing between low and high valence and arousal movies (scaled to 0 and 1 , where 0 is referring to low ratings and 1 to high ratings.

recorded the users Skin Conductance Responses (SCR) for each movie using the Shimmer2 GSR device ${ }^{2}$.

To analyse the SCR data ( 18 out of 20 valid, 2 excluded due to technical problems), we first prepared the data for the analysis by (1) using a windowing function (taking the mean of values in a widow of size 9 to smooth the data and remove imperfections, (2) standardizing the raw data for each user (values from 0 to 1), (3) reducing the frequency of data from $50 \mathrm{~Hz}$ to $20 \mathrm{~Hz}$. We then plotted all the data for each user and performed a visual analysis for each movie. All movies showed potential for the second step of the study, meaning that they all had elicited 'peak moments' (captured in the SCR responses) based on which the haptic feedback could be designed. We also took the questionnaire ratings into account in order to balance between low and high valence/arousal movies in the final selection of movies for step two. In the end, we selected six out of the 12 movies for the next step (see Table 1).

\section{Step 2: Creation of haptic feedback}

Here we describe the creation and integration of the specific mid-air haptic feedback for the six selected movies. This second step was divided into two main parts: (1) the first part is concerned with the timing of the haptic feedback and (2) the second part discusses the design of the haptic feedback (i.e. haptic pattern).

\section{Temporal integration of the haptic feedback}

In order to find the right timing for the haptic feedback (refers to the synchronisation of the haptic feedback with peak moments in a movie), a two-way manual approach was used. First, we used the SCR data (visual representation for each of the 6 selected movies, including amplitude and timing) to inform the key peak moments in the movie across users (see Figure 2). Second, we verified the 3 to 5 highest peaks revealed by the SCR data based on the narrative of the movie by comparing the timings taking into account the delay of the SCR measurements. For example, Figure 2 shows that the third peak in arousal is linked to the crocodile eating the gnu. This peak can be seen in user's SCR data at second 41, and fits the particular moment in the movie around second 39 (taking into account the 1 to 3 seconds' delay of the SCR recording). We created six synchronized haptic sequences, one for each of the short movies according to the recorded peak moments.

In addition, we create one more haptic sequence which was shared across all movies simulating an unsynchronized integration of haptic feedback. For that purpose, we defined

\footnotetext{
${ }^{2} \mathrm{http}: / / \mathrm{www}$. shimmersensing.com/
} 


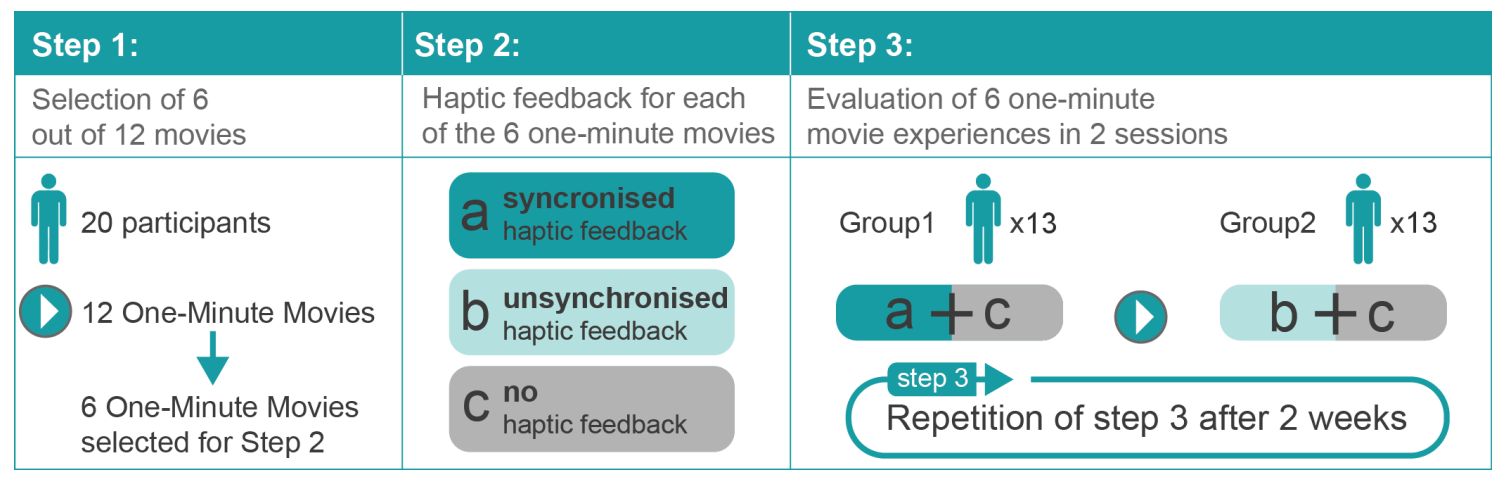

Figure 1. Overview on the study set up including the three main steps: (1) selection 6 out of 12 movies, (2) creation of the haptic feedback (i.e. haptic patterns) for the 6 selected movies, (3) evaluation of the 6 movies with and without haptic feedback in two sessions.

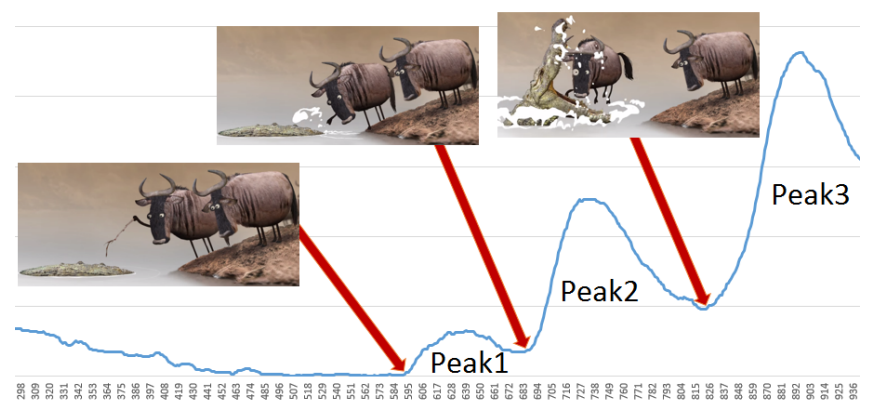

Figure 2. Example: "Wildebeest" movie. Timings and related events with the time on horizontal axis $(1$ unit $=20 \mathrm{~ms})$ and $S C R$ in vertical axis (normalized from 0 to 1).

one pattern of peak moments at second $12,32,42$ and 48 , which resemble the other creations in terms of number of peak moments and durations.

This haptic sequence is the same for all movies. Please note that there is a small possibility that the unsynchronised condition cross with the synchronisation condition, as it was nearly impossible to avoid all 6 conditions. However, we tried to keep the same sequence across all movies to show if haptics even asynchronous has an effect or not.

\section{Design of the haptic pattern}

As described in the previous section, each haptic sequence is based on a 60 seconds' timeframe and defines the timing for integrating the haptic feedback. More precisely it sets the timestamps for the design and integration of the synchronized and not synchronized (asynchronous) haptic pattern.

The mid-air haptic pattern itself consists of a single point displayed on the hand. This point changes location every $100 \mathrm{~ms}$, following a pseudo-random pattern on a five by five centimetres' square surface (similar to the feeling of rain drops on the hand, however in a dry form [22]). By using this distributed pattern, we avoid focusing on a particular part of the hand, which might be perceived either more positive or negative as previous work has shown (see [23]), and would distract the focus from the temporal integration of the haptic pattern.

The frequency of the displayed point was kept constant at $200 \mathrm{~Hz}$ and the intensity varied between $30 \%$ and $100 \%$ de- pending on pre-defined peak moments in a movie in the synchronous condition or a random time in the asynchronous condition. This design is inspired by the idea of background sound (i.e. soundtrack) which is usually present throughout a movie and increases at important moments in the movie to emphasise the emotions and immersion. Using this approach removes the surprise effect a haptic stimulus might otherwise have if it just appears at peak moments.

\section{Step 3: Viewer experience evaluation}

The aim of this evaluation step was to understand the effect of mid-air haptics on users' viewing experience. The evaluation was repeated two weeks later to account for any novelty effects of the new mid-air haptic technology used in our stud [5].

\section{Study design and methods}

For this final step in our study, we recruited 32 users. Each user experienced the final 6 movies with and without haptic feedback. One half (i.e. 16 users) received the haptic feedback synchronised with the audiovisual content (movie specific design as described in the previous section) and the other half received the unsynchronised haptic pattern which was the same across all movies (based on pre-defined fixed timestamps across all movies). The order between with and without haptic feedback was counterbalanced across users and repeated after two weeks for each user in each of the two conditions (synchronised versus unsynchronised haptic feedback).

We used a combination of measures (i.e. SAM, Liking Scale, and SCR) to capture users feedback. Users were asked to confirm that they have no sensory impairments and to complete a short demographic questionnaire (age, gender) before starting the experiment. This study was approval by the local University Ethics committee.

\section{Study set up and procedure}

For the experiment, users seated comfortably in a chair and watch the movies on a 24" computer screen. Their right hand was positioned on a custom-made armrest that was built as a box integrating the mid-air haptic device. A hole on the top indicates where users would put their palm, so that they can perceive the haptic stimulus on their hand from below.

At the beginning, we allowed users to familiarise themselves with the haptic set up and calibrated the haptic stimulus for each user: a simple focal point was displayed in the middle of 


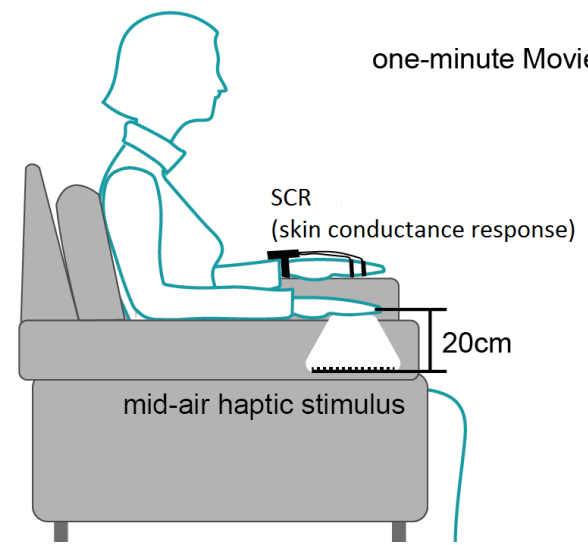

Figure 3. The study set up showing a user with on the left hand the Shimmer2 GSR device (recording the galvanic skin response) and the right hand above the mid-air haptic device.

the hole where users put their hand. The setup ensured that users kept their hand still while watching the movies (Figure 3 ). On the left hand, which was resting on arm rest, users were wearing a SCR device. Users were told not to move the left hand during the experiment and to use the right hand to answer all questionnaires (displayed on the screen between each movie).

The study itself involved a succession of six movies. However, the first movie played to each user was a 3 minutes baseline video showing a series of landscapes without any animation or sounds. During that time, SCR data was collected and used as a baseline for the SCR analysis of each user. Then the six movies were played twice, with and without haptic feedback.

Before each movie, a five second black screen was displayed to give enough time to people to put their arm back above the haptic device (right armrest) and to introduce a pause between filling in the questionnaire and starting the next movie. In order to avoid any order effects, we randomised the order of the movies using a balanced latin square of size 12 (6 movies $\times 2$ haptic conditions). After each movie, including the baseline, three main questions were asked about (1) Arousal: "How much of your emotion is activated" Self-Assessment Manikin, (2) Valence: "How did the movie made you feel?" Self-Assessment Manikin, (3) Liking: "How much did you enjoy the movie?" on the semantically Labelled Hedonic Scale (LHS) [17].

\section{Software used}

A combination of several software parts was used in the study: $\mathrm{c}++$ for programming the mid-air haptic technology, the Shimmer software for the SCR recording, and c\# for the presentation of the questionnaires and movies. All different parts - haptic feedback, movies, and SCR recording - needed to be synchronised in order to ensure the right integration and interpretation of the data. The synchronisation and timing between the software was assured by high precision internal media timers (precision $<1 \mathrm{~ms}$ ).

For the SCR recordings, we used the Shimmer 2 sensor attached to two fingers: the index and middle finger of left hand. The settings were set to $50 \mathrm{~Hz}$ for the frequency of measurement and $56 \mathrm{k} \Omega$ to $128 \mathrm{k} \Omega$ for the resistance measure.

\section{Data analysis}

The data was collapsed across all movies (the baseline movie was left out from the analysis) and a $2 \times 2 \times 2$ mixed design analysis of variance (ANOVA) with haptics (off and on) and session (first and second), and group (synchronous and asynchronous) was performed on each of the rating scales and the SCR.

The raw data of the SCR were first normalized to $20 \mathrm{~Hz}$, then an amplitude correction was applied which consisted of subtracting the lowest value recorded across movies and to all other values. Afterwards, the log of each value was calculated and the analyses were performed on these values [3].

\section{RESULTS}

The results of our analysis of the questionnaires and physiological recordings are presented in this section alongside with the users' information.

\section{Users}

In total, there were 54 users involved in all the steps of the study. Due to technical problems with the SCR recording, we removed a total of 8 users from the analysis. The prestudy involved 20 participants ( 9 female, average age 25 ), the group 1 which refer to the synchronised haptic condition (synchronised with the peak moments) involved 13 participants (4 males, average age 24.5), and the group 2 which refer to the cross-movies haptic condition (unsynchronised with the peak moments) involved 13 participants (5 males, average age 26).

\section{Questionnaires ratings}

A significant interaction $(\mathrm{p}<.05)$ between session and haptic stimulation was found for the valence ratings, and a significant main effect $(\mathrm{p}<.05)$ of haptic stimulation was found for the arousal ratings. Paired-samples t-tests performed on the interaction term failed to reveal a significant result $(\mathrm{p}=.059)$, nonetheless, the valence ratings appear to be higher in the first as compared to the second session, when the haptic stimulation was off (see Figure 4, 1A and 1B). Moreover, Bonferronicorrected comparisons revealed that the users reported feeling significantly more aroused when the haptic system was on, than when it was off $(p=.014)$. A visualization of all the mean ratings is presented in Figure 4.

\section{Skin conductance responses}

A summary of the results of the SCR is presented on Figure 5. Only a significant effect of session was found $(\mathrm{p}<.001)$. In particular, pairwise comparisons revealed that the users were more aroused in the first session than the second session. While no main effect of haptics was found, there was a small general tendency to obtain higher values when the haptic system was on $(\mathrm{M}=0.48, \mathrm{SD}=0.29)$ than when it was off $(\mathrm{M}=0.43, \mathrm{SD}=0.027)$.

\section{DISCUSSION}

We studied the possibility of augmentation of one-minute movies with mid-air haptic feedback. Our findings provide insights into how users' arousal and emotional valence are influenced by mid-air haptic stimulation, that is presented in a synchronous or asynchronous fashion alongside the movies. 


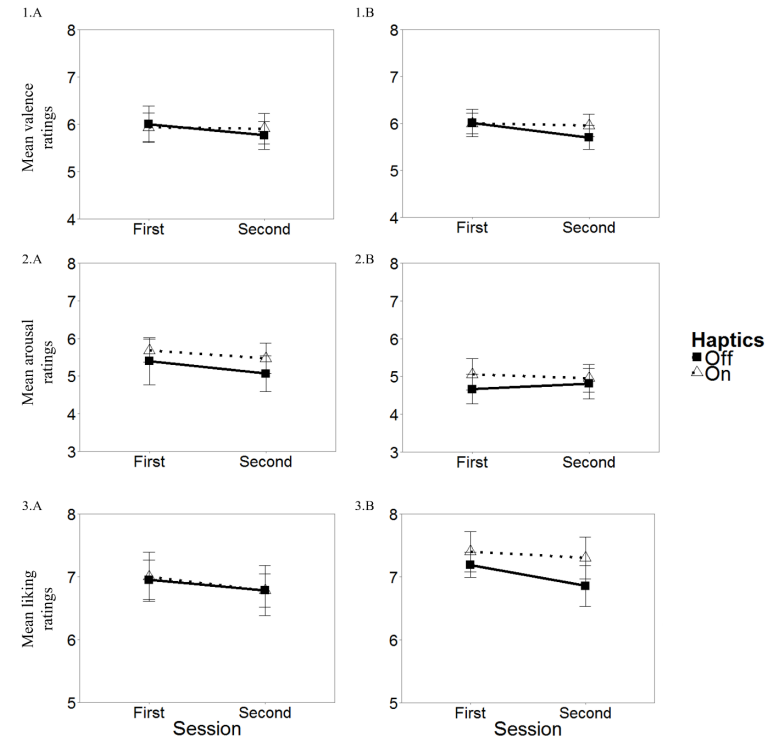

Figure 4. Summary of the questionnaire results. The numbers correspond to the different variables assessed, namely, valence (1), arousal (2), and liking (3), whilst the letters correspond to the (A) synchronous and (B) asynchronous groups. The error bars represent the standard error of the means.
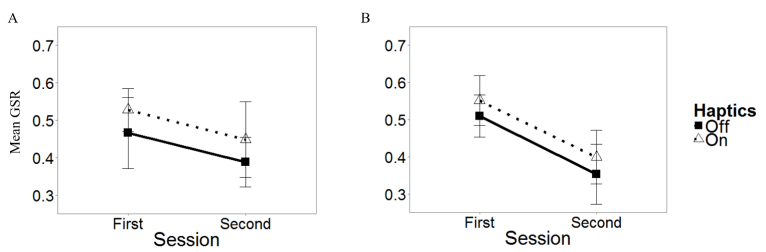

Figure 5. Summary of the SCR results. The letters correspond to the (A) synchronous and (B) asynchronous groups. The error bars represent the standard error of the means.

Below we discuss our findings and their relevance for designing haptically augmented movie experiences.

\section{Effect of mid-air haptics on first and second time viewing experiences}

Our results show that the arousal ratings are high across all conditions. This result is in line with previous work demonstrating the arousing effect of haptic feedback while watching movies $[6,16]$. While a positive effect was expected for the synchronous condition, the same effect is true for the asynchronous. In other words, even when the haptic pattern does not mimic a specific movie sequence, and is placed randomly alongside the movie, users are still more aroused than with no haptic stimulation. While this is promising in particular for the novel use of mid-air haptic feedback, it is worth noticing that based on the SCR data users' arousal is dropping during the second session in both groups. This can be explained due to the fact that users already knew the movies (familiarity), and were less excited to watch them. Moreover, the novelty effect of the device is also lowered, and yet the experience with haptics is more arousing than without.

In terms of the valence ratings, a borderline significant trend was found for the interaction between Session * Haptics (see Figure 4). Post hoc analysis failed however to reach statisti- cal significance but we observed a trend in dropped valence ratings in the second session. This might be linked to the expectation of the haptic feedback causing frustration when it is absent. Indeed, most previous work showed that adding haptic feedback to movies and and other multimedia experiences is valuable and gives a boost to the persons' experience $[16,15]$. However, its sustainability over time still needs to be verified.

\section{Effect of synchronized versus asynchronized mid-air hap- tic feedback}

No interaction was found on the synchronisation condition (temporal integration of the haptic pattern). This could be explained by the use of a specific mid-air pattern integrated at different relevant peak moments in each movie instead of designing and using a variety of patterns (e.g. making us of different spatial distributions of focal points [23], shapes [18]). Thus, the synchronization of the haptic feedback might be less evident to users, as the pattern was generic and relevant for either synchronized and unsynchronized moments in a movie.

Most previous approaches focus on synchronised feedback [15, $16,33]$ where patterns are specifically designed for a sequence. However, considering our findings, which will need further validation, it is promising that the difference between the aforesaid conditions is not significant as this gives rise to alternative design approaches, that could ultimately be simplified through providing producers and content creators with pre-defined patterns, tools to create their own patterns, or even automate the generation of haptic patterns based on the extraction of audio-visual content from a movie, as done in [32]. The synchronisation becomes less important as the emotion can be activated at different times during the sequence of a movie. Such future exploration opportunities around synchronization could become of value in relation to the MPEP-V ISO [12] standard concerned with the delivery of 'sensory information' as part of a general framework.

\section{CONCLUSIONS}

This paper provides insights into the effect of mid-air haptic feedback (a new haptic technology) on users viewing experience, specifically applied to one-minute movies. This specific content format (60 seconds narrative) allowed us to systematically investigate the design and evaluation of synchronized versus unsynchronized mid-air haptic stimuli and their effect on users perceived valence and arousal. Mid-air haptic feedback, by its ability to increase immersion, affect emotions, and contribute to the overall quality of experiences without requiring any attachment to the viewers' body, is an opportunity for interactive TV and online video. The findings are promising and open up a space for future explorations of other formats, full length movies enhanced through mid-air haptics.

\section{ACKNOWLEDGMENTS}

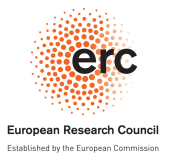

This project has received funding from the European Research Council (ERC) under the European Union's Horizon 2020 research and innovation programme under grant agreement No 638605 . 


\section{REFERENCES}

1. S. Ando and H. Shinoda. Ultrasonic emission tactile sensing. Control Systems, IEEE, 15(1):61-69, Feb 1995.

2. A. M. Baranowski and H. Hecht. The big picture: Effects of surround on immersion and size perception.

Perception, 43(10):1061, 2014.

3. W. Boucsein. Electrodermal activity. Springer Science \& Business Media, 2012.

4. M. M. Bradley and P. J. Lang. Measuring emotion: The self-assessment manikin and the semantic differential.

Journal of Behavior Therapy and Experimental Psychiatry, 25(1):49 - 59, 1994.

5. T. Carter, S. A. Seah, B. Long, B. Drinkwater, and S. Subramanian. Ultrahaptics: Multi-point mid-air haptic feedback for touch surfaces. In Proceedings of the 26th Annual ACM Symposium on User Interface Software and Technology, UIST '13, pages 505-514, New York, NY, USA, 2013. ACM.

6. F. Danieau, J. Fleureau, A. Cabec, P. Kerbiriou, P. Guillotel, N. Mollet, M. Christie, and A. Lecuyer. Framework for enhancing video viewing experience with haptic effects of motion. In Haptics Symposium (HAPTICS), 2012 IEEE, pages 541-546, March 2012.

7. M. Faust and Y.-H. Yoo. Haptic feedback in pervasive games. In Third International Workshop on Pervasive Gaming Applications, volume 7, pages 1-8, 2006.

8. G. Ghinea, C. Timmerer, W. Lin, and S. R. Gulliver. Mulsemedia: State of the art, perspectives, and challenges. ACM Trans. Multimedia Comput. Commun. Appl., 11(1s):17:1-17:23, Oct. 2014.

9. D. Hecht and M. Reiner. Sensory dominance in combinations of audio, visual and haptic stimuli. Experimental brain research, 193(2):307-314, 2009.

10. M. J. Hertenstein, R. Holmes, M. McCullough, and D. Keltner. The communication of emotion via touch. Emotion, 9(4):566-573, 2009.

11. S. Hwang and J. hee Ryu. The haptic steering wheel: Vibro-tactile based navigation for the driving environment. In 2010 8th IEEE International Conference on Pervasive Computing and Communications Workshops (PERCOM Workshops), pages 660-665, March 2010.

12. Information technology - Media context and control. Standard, International Organization for Standardization, Geneva, CH, Jan. 2014.

13. A. Israr, S.-C. Kim, J. Stec, and I. Poupyrev. Surround haptics: Tactile feedback for immersive gaming experiences. In CHI' 12 Extended Abstracts on Human Factors in Computing Systems, CHI EA '12, pages 1087-1090, New York, NY, USA, 2012. ACM.

14. A. Israr, S. Zhao, K. Schwalje, R. Klatzky, and J. Lehman. Feel effects: Enriching storytelling with haptic feedback. ACM Trans. Appl. Percept., 11(3):11:1-11:17, Sept. 2014.
15. Y. Kim, J. Cha, I. Oakley, and J. Ryu. Exploring tactile movies: An initial tactile glove design and concept evaluation. IEEE MultiMedia, PP(99):1-1, 2015.

16. P. Lemmens, F. Crompvoets, D. Brokken, J. van den Eerenbeemd, and G.-J. de Vries. A body-conforming tactile jacket to enrich movie viewing. In EuroHaptics conference, 2009 and Symposium on Haptic Interfaces for Virtual Environment and Teleoperator Systems. World Haptics 2009. Third Joint, pages 7-12, New York, NY, USA, March 2009. IEEE.

17. J. Lim, A. Wood, and B. G. Green. Derivation and evaluation of a labeled hedonic scale. Chemical senses, 34(9):739-751, 2009.

18. B. Long, S. A. Seah, T. Carter, and S. Subramanian. Rendering volumetric haptic shapes in mid-air using ultrasound. ACM Trans. Graph., 33(6):181:1-181:10, Nov. 2014.

19. R. B. Lull and B. J. Bushman. Immersed in violence: Presence mediates the effect of $3 \mathrm{~d}$ violent video gameplay on angry feelings. 2014.

20. N. Murray, B. Lee, Y. Qiao, and G.-M. Muntean. Olfaction-enhanced multimedia: A survey of application domains, displays, and research challenges. ACM Comput. Surv., 48(4):56:1-56:34, May 2016.

21. H. Nakamura and H. Miyashita. Augmented gustation using electricity. In Proceedings of the $2 \mathrm{Nd}$ Augmented Human International Conference, $\mathrm{AH}$ ' 11, pages 34:1-34:2, New York, NY, USA, 2011. ACM.

22. M. Obrist, S. A. Seah, and S. Subramanian. Talking about tactile experiences. In Proceedings of the SIGCHI Conference on Human Factors in Computing Systems, CHI '13, pages 1659-1668, New York, NY, USA, 2013. ACM.

23. M. Obrist, S. Subramanian, E. Gatti, B. Long, and T. Carter. Emotions mediated through mid-air haptics. In Proceedings of the 33rd Annual ACM Conference on Human Factors in Computing Systems, CHI '15, pages 2053-2062, New York, NY, USA, 2015. ACM.

24. M. Obrist, C. Velasco, C. Vi, N. Ranasinghe, A. Israr, A. Cheok, C. Spence, and P. Gopalakrishnakone. Sensing the future of hci: Touch, taste, and smell user interfaces. interactions, 23(5):40-49, Aug. 2016.

25. M. Obrist, C. Velasco, C. T. Vi, N. Ranasinghe, A. Israr, A. D. Cheok, C. Spence, and P. Gopalakrishnakone. Touch, taste, \&\#38; smell user interfaces: The future of multisensory hci. In Proceedings of the 2016 CHI Conference Extended Abstracts on Human Factors in Computing Systems, CHI EA '16, pages 3285-3292, New York, NY, USA, 2016. ACM.

26. N. Ranasinghe and E. Y.-L. Do. Digital lollipop: Studying electrical stimulation on the human tongue to simulate taste sensations. ACM Transactions on Multimedia Computing, Communications, and Applications (TOMM), 13(1):5, 2016. 
27. O. S. Schneider, A. Israr, and K. E. MacLean. Tactile animation by direct manipulation of grid displays. In Proceedings of the 28th Annual ACM Symposium on User Interface Software \&\#38; Technology, UIST '15, pages 21-30, New York, NY, USA, 2015. ACM.

28. R. Sodhi, I. Poupyrev, M. Glisson, and A. Israr. Aireal: Interactive tactile experiences in free air. ACM Trans. Graph., 32(4):134:1-134:10, July 2013.

29. C. Timmerer, M. Waltl, B. Rainer, and N. Murray. Sensory Experience: Quality of Experience Beyond Audio-Visual, pages 351-365. Springer International Publishing, Cham, 2014.

30. S. u. Rehman, J. Sun, L. Liu, and H. Li. Turn your mobile into the ball: Rendering live football game using vibration. IEEE Transactions on Multimedia, 10(6):1022-1033, Oct 2008.

31. G. Wilson, T. Carter, S. Subramanian, and S. A. Brewster. Perception of ultrasonic haptic feedback on the hand: Localisation and apparent motion. In Proceedings of the
SIGCHI Conference on Human Factors in Computing Systems, CHI '14, pages 1133-1142, New York, NY, USA, 2014. ACM.

32. Y.-H. Yang, Y.-C. Lin, Y.-F. Su, and H. H. Chen. A regression approach to music emotion recognition. IEEE Transactions on audio, speech, and language processing, 16(2):448-457, 2008.

33. Z. Yuan, G. Ghinea, and G. M. Muntean. Quality of experience study for multiple sensorial media delivery. In 2014 International Wireless Communications and Mobile Computing Conference (IWCMC), pages 1142-1146, Aug 2014.

34. S. Zhao, O. Schneider, R. Klatzky, J. Lehman, and A. Israr. Feelcraft: Crafting tactile experiences for media using a feel effect library. In Proceedings of the Adjunct Publication of the 27th Annual ACM Symposium on User Interface Software and Technology, UIST'14 Adjunct, pages 51-52, New York, NY, USA, 2014. ACM. 